\title{
Learning Strategies for Knowledge-Base Updating in Online Signature Verification Systems
}

\author{
Giuseppe Pirlo ${ }^{1}$, Donato Impedovo ${ }^{2}$, and Donato Barbuzzi ${ }^{1}$ \\ ${ }^{1}$ Department of Computer Science, University of Bari, Bari, Italy \\ \{giuseppe.pirlo, donato.barbuzzi\}@uniba.it \\ ${ }^{2}$ Department of Electrical and Electronic Engineering, Polytechnic of Bari, Bari, Italy \\ impedovo@deemail.poliba.it
}

\begin{abstract}
Updating of reference information is a crucial task for automatic signature verification. In fact, signature characteristics vary in time and whatever approach is considered the effectiveness of a signature verification system strongly depends on the extent to which reference information is able to model the changeable characteristics of users' signatures. This paper addresses the problem of knowledge-base updating in multi-expert signature verification systems and introduces a new strategy which exploits the collective behavior of classifiers to select the most profitable samples for knowledge-base updating. The experimental tests, carried out using the SUSig database, demonstrate the effectiveness of the new strategy.
\end{abstract}

Keywords: Signature Verification, Feedback-based Strategy, Multi Expert System.

\section{Introduction}

Biometrics is an emerging field of research and technology that involves the recognition of individuals through their physical or behavioural traits. Examples of physical attributes are fingerprints, facial features, the iris, DNA, etc. Some behavioural characteristics are the signature, the voice and keystroke dynamics, among others $[1,2,3]$.

A biometric system can either verify or identify. In verification mode, it authenticates the person's identity on the basis of his/her claimed identity. Instead, in identification mode, it establishes the person's identity (among those enrolled in a database) without the subjects having to claim their identity $[4,5]$.

Signature verification occupies a very special place in biometrics. A signature is a biometric trait generated by a complex process originating in the signer's brain as a motor control "program", implemented through the neuromuscular system and left on the writing surface by a handwriting device $[6,7]$. The three main phases of automatic signature verification are: data acquisition and preprocessing, feature extraction and classification [3]. During enrolment phase, the input signatures are processed and their personal features are extracted and stored into knowledge base. During the classification phase, personal features extracted from an inputted signature are compared 
against the information in the knowledge base, in order to judge the authenticity of the inputted signature [8].

Recently, it has been observed that the verification accuracy can be also improved combining multiple experts. The idea is not to rely on a single decision making scheme but to use several designs (experts) for decision making [9, 10]. In fact, the collective behavior of a set of classifiers can convey more information that those of each classifier of the set, and this information can be exploited for classification aims $[11,12]$. When multiple experts are considered, the collective behavior of the set of individual experts can be exploited to dynamically update the knowledge-base of the system, according to suitable feed-back based strategies. In fact, a handwritten signatures is the result of a complex process depending on the psychophysical state of the signer and the conditions under which the signature apposition process occurs. Therefore, although complex theories have been proposed to model the psychophysical mechanisms underlying handwriting [6,7], ink-depository processes [13] and regional distribution of information $[14,15,16]$, signature verification still remains an open challenge since a signature is judge to be genuine or a forgery only on the basis of a few reference specimens.

This paper proposes, as Figure 1 shows, the selection of the valuable samples useful to update the knowledge-base of all experts of the set, correctly recognized by the multi-expert system.

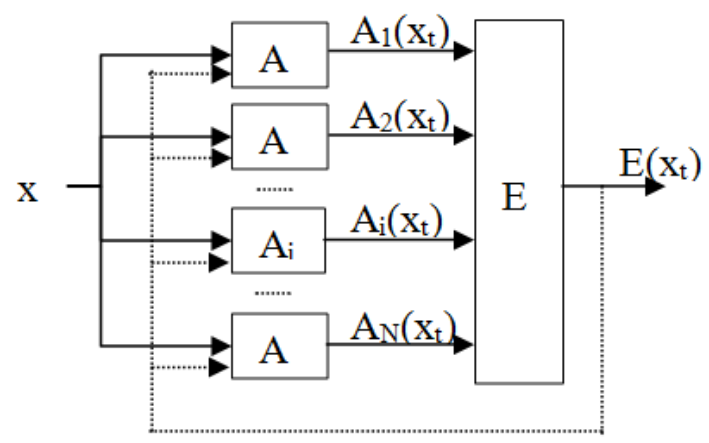

Fig. 1. Feedback in the Multi-Expert Parallel System

Tests have been performed on the task of automatic signature verification, on the SUSig database by considering different types of features. Two different combination techniques (Majority Vote, Weighted Majority vote) have been used at abstract level. The results demonstrate that the proposed strategy is effective in terms of reduction of the false rejection rate (FRR) of genuine signatures and of the false acceptance rate (FAR) of forgery signatures.

The paper is organized as follows: Section 2 presents the background of retraining rules and the different feedback-based strategies. Operating conditions and results are, respectively, in Section 3 and 4. Section 5 reports a discussion and the conclusion of the work. 


\section{Background of Retraining Rules}

\subsection{Related Work}

In literature, several methods have been proposed for solving the retraining classifier issue, when new labeled data became available. So, the simplest way to update the knowledge base of classifier is probably to use the entire new dataset to retrain the system given the initial training condition or, depending by the classifier, the sets of new and old data. On the other hand, many interesting algorithms can be adopted in order to select (or focus the attention on) specific samples. In particular, the algorithm AdaBoost $[17,18]$ is able to improve performance of a classifier on a given data set by focusing the learner attention on difficult instances. Even if this approach is very powerful, it works well in the case of weak classifiers, moreover not all the learning algorithms accept weights for the incoming samples. Another interesting approach is the bagging one: a number of weak classifiers trained on different subset (random instance) of the entire dataset are combined by means of the simple majority voting [19]. Bagging and AdaBoost algorithms are adapted when considering a single classifier but applied to a ME system, them performance are boosted. Finally, it has been observed that the collective behavior of classifiers can be considered to select the most profitable samples in order to update the knowledge base of classifiers.

In the past, samples to be used for retraining were selected by considering those, misclassified by a specific expert of the set, which produced a misclassification at the ME level [20, 21, 22].

In the next paragraph a new strategy is depicted taking into account of a multiexpert system that works in supervised learning.

\subsection{Selecting Instances}

Let be:

- $C_{j}$, for $\mathrm{j}=1,2, \ldots, \mathrm{M}$, the set of pattern classes;

- $P=\left\{x_{k} \mid k=1,2, \ldots, K\right\}$, a set of pattern to be feed to the Multi Expert (ME) system. $\mathrm{P}$ is considered to be partitioned into $\mathrm{S}$ subsets $\mathrm{P}_{1}, \mathrm{P}_{2}, \ldots, \mathrm{P}_{\mathrm{s}}, \ldots, \mathrm{P}_{\mathrm{S}}$, being $P_{s}=\left\{x_{k} \in P \mid k \in\left[N_{s} \cdot(s-1)+1, N_{s} \cdot s\right]\right\}$ and $N_{s}=K / S\left(N_{s}\right.$ integer $)$, that are fed to the multiexpert system. In particular, $\mathrm{P}_{1}$ is used for learning only, whereas $\mathrm{P}_{2}, \mathrm{P}_{3}, \ldots, \mathrm{P}_{\mathrm{s}}, \ldots, \mathrm{P}_{\mathrm{S}}$ are used both for classification and learning (when necessary);

- $y_{s} \in \Omega$, the label for the $x_{s}$ pattern, $\Omega=\left\{C_{1}, C_{2}, \ldots, C_{M}\right\}$;

- $A_{i}$ the $i$-th classifier for $\mathrm{i}=1,2, \ldots, \mathrm{N}$;

- $\mathrm{F}_{\mathrm{i}}(\mathrm{k})=\left(\mathrm{F}_{\mathrm{i}, 1}(\mathrm{k}), \mathrm{F}_{\mathrm{i}, 2}(\mathrm{k}), \ldots, \mathrm{F}_{\mathrm{i}, \mathrm{r}}(\mathrm{k}), \ldots, \mathrm{F}_{\mathrm{i}, \mathrm{R}}(\mathrm{k})\right)$ the feature vector used by $\mathrm{A}_{\mathrm{i}}$ for representing the pattern $x_{k} \in P$ (for the sake of simplicity it is here assumed that each classifier uses $\mathrm{R}$ real values as features); 
- $K B_{i}(k)$, the knowledge base of $A_{i}$ after the processing of $P_{k}$. In particular $K B_{i}(k)=\left(K B_{i}^{1}(k), K B_{i}^{2}(k), \ldots, K B_{i}^{M}(k)\right)$;

- $E$ the multi expert system which combines $H_{i}$ hypothesis in order to obtain the final one.

In first stage $(s=1)$, the classifier $\mathrm{A}_{\mathrm{i}}$ is trained using the patterns $\mathrm{x}_{\mathrm{k}} \in \mathrm{P}_{\mathrm{i}}{ }_{\mathrm{i}}=\mathrm{P}_{1}$. Therefore, the knowledge base $\mathrm{KB}_{\mathrm{i}}(\mathrm{s})$ of $\mathrm{A}_{\mathrm{i}}$ is initially defined as:

$$
\mathrm{KB}_{\mathrm{i}}(\mathrm{s})=\left(\mathrm{KB}^{1}{ }_{\mathrm{i}}(\mathrm{s}), \mathrm{KB}^{2}{ }_{\mathrm{i}}(\mathrm{s}), \ldots, \mathrm{KB}^{\mathrm{j}}{ }_{\mathrm{i}}(\mathrm{s}), \ldots, \mathrm{KB}^{\mathrm{M}}{ }_{\mathrm{i}}(\mathrm{s})\right)
$$

where, for $\mathrm{j}=1,2, \ldots, \mathrm{M}$ :

$$
\mathrm{KB}^{\mathrm{j}}{ }_{\mathrm{i}}(\mathrm{s})=\left(\mathrm{F}_{\mathrm{i}, 1}^{\mathrm{j}}(\mathrm{s}), \mathrm{F}_{\mathrm{i}, 2}^{\mathrm{j}}(\mathrm{s}), \ldots, \mathrm{F}_{\mathrm{i}, \mathrm{r}}^{\mathrm{j}}(\mathrm{s}), \ldots, \mathrm{F}_{\mathrm{i}, \mathrm{R}}^{\mathrm{j}}(\mathrm{s})\right)
$$

being $\mathrm{F}_{\mathrm{i}, \mathrm{r}} \mathrm{s}$ ) the set of the $r$-th feature of the $i$-th classifier for the patterns of the class $\mathrm{C}_{\mathrm{j}}$ that belongs to $\mathrm{P}_{\mathrm{i}}{ }_{\text {. }}$.

Successively, the subsets $\mathrm{P}_{2}, \mathrm{P}_{3}, \ldots, \mathrm{P}_{\mathrm{s}}, \ldots, \mathrm{P}_{\mathrm{S}}$ is provided to the multi-classifier system both for classification and for learning. The "leave-one-out" method is used to test the multi-expert system. When considering new labeled data (samples of $\mathrm{P}_{2}, \mathrm{P}_{3}, \ldots, \mathrm{P}_{\mathrm{s}}, \ldots, \mathrm{P}_{\mathrm{s}}$ ), a naïve and not naïve strategy can be used.

The naïve strategy uses all the available new patterns to update the knowledge base of each individual classifier:

- $\forall x_{t} \in P_{s}:$ update_KB

The second approach is derived from AdaBoost and bagging. $A_{i}$ is updated by considering all its samples correctly recognized by ME system:

- $\forall x_{t} \in P_{s} \ni^{\prime}\left(E\left(x_{t}\right)=y_{t}\right):$ update_KB

In order to inspect and take advantage of the common behavior of the ensemble of classifiers, the following simple strategy is evaluated and compared to the previous.

\section{Operating Conditions}

\subsection{Classifier and Combination Techniques}

The classifier used for the experimentation is a Naïve Bayes Classifier. This fits, in the training phase, a multivariate normal density to each class $\mathrm{Cj}$ by considering a diagonal covariance matrix. Given an input to be classified, the Maximum a Posteriori (MAP) decision rule is adopted to select the most probable hypothesis among the different classes.

Also many approaches have been considered so far for classifiers combination. These approaches differ in terms of type of output they combine, system topology and degree of a-priori knowledge they use [9, 10]. The combination technique plays a crucial role in the selection of new patterns to be feed to the classifier in the proposed 
approach. In this work the following decision combination techniques have been considered and compared: Majority Vote (MV) and Weighted Majority Vote (WMV). MV just considers labels provided by the individual classifiers, it is generally adopted if no knowledge is available about performance of classifiers so that they are equalconsidered. The second approach can be adopted by considering weights related to the performance of individual classifiers on a specific dataset. Given the case depicted in this work, it seems to be more realistic, in fact the behavior of classifiers can be evaluated, for instance, on the new available dataset. In particular, let $\mathcal{E}_{i}$ be the error rate of the i-th classifier evaluated on the last available training set, the weight assigned to

$$
A_{i} \text { is, } w_{i}=\log \left(1 / \beta_{i}\right) \text { being } \beta_{i}=\varepsilon_{i} /\left(1-\varepsilon_{i}\right)
$$

\subsection{SUSig Handwritten Signature Database}

The SUSig database consists of online signatures donated by 100 people (29 women and 71 men) [23]. The database was collected in two separate sessions that were approximately one week apart. Each person supplied 10 samples of his/her regular signature in each session, for a total of 20 genuine signatures, without any constraints on how to sign. Each person was then asked to forge a randomly selected user's signature. So, 10 forged signatures were produced.

Therefore, in our case a multi-expert system for on-line signature verification has been considered $P=\{x j \mid j=1,2, \ldots, 30\}$ (classes " 0 " and " 1 ") has been used. Figure 2 presents some samples of handwritten signatures [23].

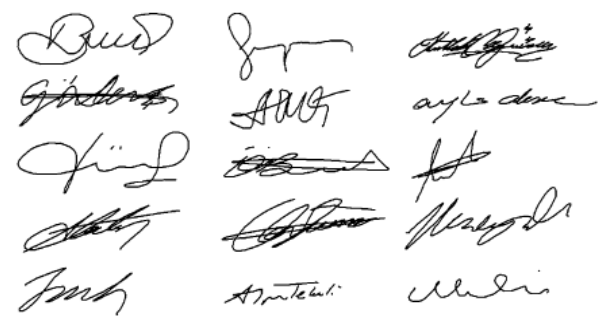

Fig. 2. Sample genuine signature from SUSig

The DB has been initially partitioned into 2 subsets:

- $\mathrm{P}_{1}=\left\{\mathrm{x}_{1}, \ldots, \mathrm{x}_{8}, \mathrm{x}_{11}, \ldots, \mathrm{x}_{18}, \mathrm{x}_{21}, \ldots, \mathrm{x}_{28}\right\}$,

- $\mathrm{P}_{2}=\left\{\mathrm{x}_{9}, \mathrm{X}_{10}, \mathrm{x}_{19}, \mathrm{x}_{20}, \mathrm{x}_{29}, \mathrm{x}_{30}\right\}$.

In particular, $\mathrm{P}_{1}$ represents the set usually adopted for training and test considering the "leave-one-out" method on SUSig DB [21]. $\mathrm{P}_{2}$ is the feedback dataset. Each signature is partitioned into 5 stroke, successively, for each stroke, the following set of features have been considered: 
- $\mathrm{F}_{1}$ : features set 1: mean and variance of the distances between two consecutive points;

- $\mathrm{F}_{2}$ : features set 2: mean and variance of pressure between two consecutive points;

- $\mathrm{F}_{3}$ : features set 3: mean and variance of the velocity and acceleration between two consecutive points.

\section{$4 \quad$ Results}

This section presents the results in terms of false rejection rate (FRR) of genuine signatures and the false acceptance rate (FAR) of forgery signatures. We combined, adopting a multi-expert system, the three set of features (F1, F2 and F3) and a classifier NB. The label "X-feed" refers to the use of the X modality for the feedback training process: "All" is the feedback of the entire set. "MV" and "WMV" are feedback at ME level adopting, respectively, the majority vote and the weighted majority vote schema.

Tables 1 show results related to the use of NB classifier and majority vote as combination technique. P1 is used for training and test in "leave-one-out" approach and P2 is used for feedback learning. The first column (No-feed) reports results related to the use of P1 for training and test, without applying any feedback (0 Selected Samples), while the approach All-feed uses all samples belonging to the new set in order to update the knowledge base of each single classifier (All Selected Samples). For MV-feed an improvement both of the FAR is $1.36 \%$ and of the FRR is $0.59 \%$ respect to the use of the entire new dataset.

Table 1. NB, MV Combination Technique, Feedback $-\mathrm{P}_{2}$

\begin{tabular}{cccc}
\hline & No-feed & MV-feed & All-feed \\
\hline FAR & 5.39 & $\mathbf{3 . 6 9}$ & 5.05 \\
FRR & 6.25 & $\mathbf{5 . 5 8}$ & 6.17 \\
\hline
\end{tabular}

In particular, figure 3 shows that MV-feed outperforms each other feedback-based strategy.

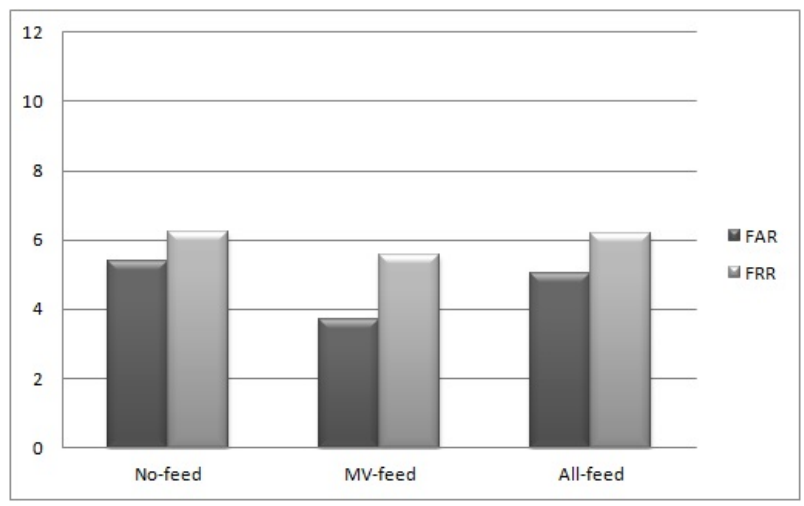

Fig. 3. Comparison between different feedback-based strategies: MV Combination 
Finally, Tables 2 show results related to the use of NB classifier and weighted majority vote as combination technique. P1 is used for training and test in "leave-oneout" approach and P2 is used for feedback learning. In this case, for WMV-feed an improvement both of the FAR is $0.68 \%$ and of the FRR is $1.32 \%$ compared to the use of the All-feed strategy where entire new dataset is used for the feedback. More specifically, figure 4 shows that the results provided by the WMV-feed approach are superior to those obtained by All-feed.

Table 2. NB, WMV Combination Technique, Feedback $-\mathrm{P}_{2}$

\begin{tabular}{lccc}
\hline & No-feed & WMV-feed & All-feed \\
\hline FAR & 5.19 & $\mathbf{2 . 9 5}$ & 3.63 \\
FRR & 9.57 & $\mathbf{5 . 8 1}$ & 7.13 \\
\hline
\end{tabular}

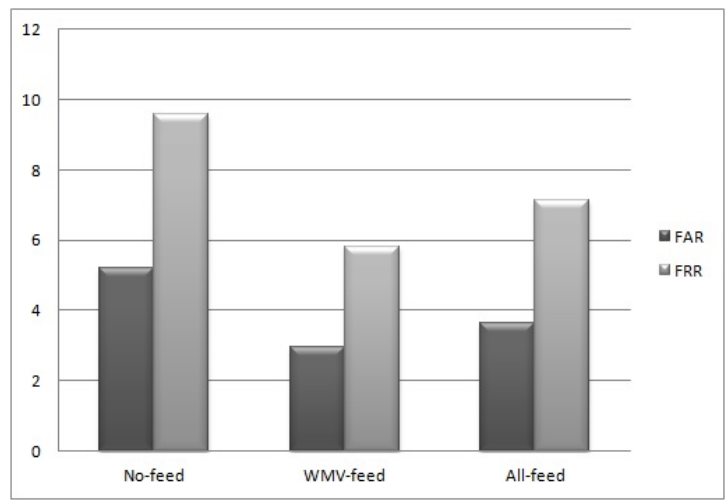

Fig. 4. Comparison between different feedback-based strategies. WMV Combination.

\section{$5 \quad$ Discussion and Conclusion}

This paper shows the possibility to improve the effectiveness of a multi-expert system for automatic signature verification and presents a new strategy which exploits the collective behavior of classifiers to select the most profitable samples for knowledgebase updating.

The experimental results demonstrate the collective behavior of a set of classifiers provides useful information to improve system performance, depending on the feature type and matching strategy. Future work will inspect these issues.

\section{References}

1. Boyer, K.W., Govindaraju, V., Ratha, N.K.: Special issue on recent advances in biometric systems. IEEE T-SMC - Part B 37(5), 1091-1095 (2007) 
2. Prabhakar, S., Kittler, J., Maltoni, D., O'Gorman, L., Tan, T.: Special issue on biometrics: Progress and directions. IEEE T-PAMI 29(4), 513-516 (2007)

3. Impedovo, D., Pirlo, G.: Automatic Signature Verification - State of the Art. IEEE Transactions on Systems, Man and Cybernetics - Part C: Applications and Review 38(5), 609-635 (2008)

4. Jain, A.K., Hong, L., Pankanti, S.: Biometric identification. Commun. ACM 43(2), 91-98 (2000)

5. Jain, A.K., Flynn, P., Ross, A.: Handbook of Biometrics. Springer, NewYork (2007)

6. Plamondon, R.: A kinematic theory of rapid human movements: Part I: Movement representation and generation. Biol. Cybern. 72(4), 295-307 (1995)

7. Plamondon, R.: A kinematic theory of rapid human movements: Part III: Kinetic outcomes. Biol. Cybern. (1997)

8. Impedovo, D., Pirlo, G., Plamondon, R.: Handwritten Signature Verification: New Advancements and Open Issues. In: Proc. XIII International Conference on Frontiers in Handwriting Recognition (ICFHR 2012), Monopoli, Bari, Italy, pp. 18-20, 365-370 (2012)

9. Kittler, J., Hatef, M., Duin, R.P.W., Matias, J.: On combining classifiers. IEEE Trans. on PAMI 20(3), 226-239 (1998)

10. Di Lecce, V., Dimauro, G., Guerriero, A., Impedovo, S., Pirlo, G., Salzo, A.: A Multiexpert System for Dynamic Signature Verification. In: Kittler, J., Roli, F. (eds.) MCS 2000. LNCS, vol. 1857, pp. 320-329. Springer, Heidelberg (2000)

11. Barbuzzi, D., Impedovo, D., Pirlo, G.: Supervised Learning Strategies in Multi-Classifier Systems. In: Proc. 11th International Conference on Information Science, Signal Processing and their Applications (ISSPA 2012), Montreal, Canada, July 3-5, pp. 1215-1220 (2012)

12. Pirlo, G., Trullo, C.A., Impedovo, D.: A Feedback-based multi-classifier system. In: Proceedings of the 10th International Conference on Document Analysis and Recognition (ICDAR-10), Barcelona, Spain, July 26-29, pp. 713-717. IEEE Computer Society Press (2009)

13. Franke, K., Rose, S.: Ink-deposition analysis using temporal data. In: Proc. 10th Int. Workshop Front. Handwriting Recognition (IWFHR), La Baule, France, pp. 447-453 (2006)

14. Pirlo, G., Impedovo, D.: Fuzzy-Zoning-Based Classification for Handwritten Characters. IEEE Trans. on Fuzzy Systems 19(4), 780-785 (2011)

15. Impedovo, S., Ferrante, A., Modugno, R., Pirlo, G.: Feature Membership Functions in Voronoi-based Zoning. In: Serra, R., Cucchiara, R. (eds.) AI*IA 2009. LNCS, vol. 5883, pp. 202-211. Springer, Heidelberg (2009)

16. Impedovo, S., Modugno, R., Ferrante, A., Pirlo, G.: Zoning Methods for Hand-written Character Recognition: An Overview. In: Proceedings of the 12th International Conference on Frontiers in Handwriting Recognition (ICFHR-12), Kolkata, India, November 16-18, pp. 329-334. IEEE Computer Society Press (2010)

17. Schapire, R.E.: The strength of weak learnability. Machine Learning 5(2), 197-227 (1990)

18. Polikar, R.: Bootstrap-Inspired Techniques in Computational Intelligence. IEEE Signal Processing Magazine 24(4), 59-72 (2007)

19. Freud, Y., Schapire, R.E.: Decision-theoretic generalization of on-line learning and an application to boosting. J. of Comp. and System Sciences 55(1), 119-139 (1997)

20. Impedovo, D., Pirlo, G.: Updating Knowledge in Feedback-based Multi-Classifier Systems. In: Proc. of ICDAR, pp. 227-231 (2011) 
21. Barbuzzi, D., Impedovo, D., Pirlo, G.: Feedback-Based Strategies In Multi-Expert Systems. In: Sesto Convegno del Gruppo Italiano Ricercatori in Pattern Recognition (2012)

22. Barbuzzi, D., Impedovo, D., Pirlo, G.: Benchmarking of Update Learning Strategies on Digit Classifier Systems. In: Proceedings of the 13th International Conference on Frontiers in Handwriting Recognition, pp. 35-40 (2012)

23. Kholmatov, A., Yanikoglu, B.: SUSIG: an on-line signature database, associated protocols and benchmark results. Pattern Analysis and Applications, 1-10 (2008) 